\title{
Comparison of Surgical and Functional Outcome of Laparoscopic Pyeloplasty and Robot-assisted Pyeloplasty for Congenital Uretero Pelvic Junction Obstruction
}

\author{
(1) Aditya Abhishek Jha1, (1) Arjun Singh Sandhu², (1) Sharat Chandra Dash33, (1) Raghav Talwar', (1) Madhu Govindaiah4, \\ (D) Gagandeep Singh5, (D) Anoop Handa33, (D) Nimit Solanki2 \\ 1 Department of Urology, Military Hospital, Secunderabad, Telangana, India \\ 2Department of Urology, Base Hospital Delhi Cantt, New Delhi, India \\ ${ }^{3}$ Department of Urology, Army Hospital Research and Referral, New Delhi, India \\ ${ }^{4}$ Department of Urology, Command Hospital Air Force, Bengaluru, Karnataka, India \\ 5 Department of Urology, Command Hospital, Udhampur, Jammu and Kashmir, India
}

\section{What's known on the subject? and What does the study add?}

Most of the studies done in the past show that with all other intraoperative and postoperative parameters being comparable between robotic and laparoscopic pyeloplasty, only total operative time and total blood loss happen to be both clinically and statistically significantly lesser in robotic pyeloplasty. Our study shows that in the experienced hand, only total operative time happens to be clinically and statistically significantly lesser in robotic pyeloplasty whereas, total blood loss is not clinically significantly lesser.

\section{Abstract}

Objective: A surge in the easy availability of robotic platforms has resulted in numerous surgical procedures, which were previously done using an open or conventional laparoscopic approach, are now being done using robots worldwide. A prospective randomized study was conducted to compare surgical and functional outcomes of conventional laparoscopic pyeloplasty with robotic-assisted pyeloplasty.

Materials and Methods: Patients who require pyeloplasty who presented to our institute between June 2015 and March 2018 were randomized into a robot-assisted or conventional laparoscopic pyeloplasty group. Common steps included a lateral trans-peritoneal approach, intraoperative antegrade double-J stent placement, stent removal at 4 weeks postoperative, and Diethylene Triamine Penta Acetate renogram at 4 weeks post stent removal. Records of intraoperative and postoperative variables were maintained for all patients. The comparison of continuous numerical data was done using the Independent t-test and categorical non-numerical data using the chi-square $\left(\chi^{2}\right)$ test. P-values of $<0.05$ were considered significant.

Results: This study includes 58 patients who were randomized into two groups with 29 patients each. No significant difference was noted for postoperative variables, such as the visual analog score for pain, drain placement duration, hospitalization duration, and time to return to daily activity. Intraoperative variables, such as total operative time (148.56 minute vs. 114.28 minute, $p$-value=0.001) and intraoperative blood loss (68.4 $\mathrm{mL}$ vs. $59.2 \mathrm{~mL}$, p-value=0.001) were significantly lesser and favored robot-assisted pyeloplasty over conventional laparoscopic pyeloplasty.

Conclusion: In favor of robot-assisted pyeloplasty, both statistically and clinically intraoperative time was lesser, but intraoperative blood loss was lesser only statically and not clinically.

Keywords: Laparascopic pyeloplasty, robotic pyeloplasty, congenital ureteropelvic junction obstruction

Correspondence: Anoop Handa MD, Department of Urology, Army Hospital Research and Referral, New Delhi, India

E-mail: dr.anoop10@gmail.com ORCID-ID: orcid.org/0000-0003-2364-7854

Received: 09.12.2020

Accepted: 02.12 .2021

Cite this article as: Jha AA, Sandhu AS, Dash SC, Talwar R, Govindaiah M, Singh G, Handa A, Solanki N. Comparison of Surgical and Functional Outcome of Laparoscopic Pyeloplasty and Robot-assisted Pyeloplasty for Congenital Uretero Pelvic Junction Obstruction. J Urol Surg, 2022;9(1):20-24.

oCopyright 2022 by the Association of Urological Surgery / Journal of Urological Surgery published by Galenos Publishing House. 


\section{Introduction}

The management of ureteric pelvic junction obstruction (UPJO) has dramatically evolved over the past two decades. Laparoscopy has largely replaced open pyeloplasty and has become the standard of care for primary UPJO $(1,2)$. However, conventional laparoscopic pyeloplasty (CLP) remains a technically demanding procedure that requires advanced intracorporeal suturing skills (3).

The availability of robotic platforms has altered the way urologists approach various complex reconstructive procedures. The need for precise intracorporeal suturing makes pyeloplasty a likely procedure, which would benefit from robotic assistance. Therefore, this randomized study aimed to compare functional and surgical outcomes following CLP and robot-assisted laparoscopic pyeloplasty (RALP) to explore this issue.

\section{Materials and Methods}

The study was conducted between June 2015 and Mar 2018 in a tertiary care center in Northern India. All patients of congenital UPJO presenting with pain, recurrent urinary tract infection, secondary renal calculus, or deteriorating renal function were randomized and recruited. Patients with secondary/recurrent UPJ0 were excluded from the study. The primary objective was to compare the success rate of the procedures between the two groups by demonstrating non-obstructed drainage and postoperative symptom resolution, with secondary objectives to compare surgical parameters like total operative time, total blood loss, intraoperative complications, postoperative pain, and durations of drain placement, hospitalization, and return to daily activity.

\section{Statistical Analysis}

Descriptive statistics were used to characterize the demographic data, laterality of obstruction (right/left), indication of surgery (pain, incidental detected), intraoperative finding, complications and success of surgery. Mean and standard deviation was used for quantitative continuous variables. Categorical non numerical data analysis was done with chi square test. The $p$ value of $<0.05$ was considered statistically significant.

Catering to an alpha error of 5\% and power of study as $80 \%$, a sample size of 50 cases ( 25 cases in each group) was calculated. The feasibility of $10 \%$ lost to follow-up was factored in, thus we enrolled 58 cases in total (29 cases each group).

This single-center prospective randomized controlled trial randomized patients (1:1) using computer-generated random numbers into the two groups. Random numbers were generated using the RAND function of MS Excel. They were divided into two equal groups. The results were kept in serially numbered, sealed, opaque envelopes. These envelopes were kept with a third person. Once the patient was enrolled, a call was given to the third person to ascertain the group. Both surgeon and patient were informed about the technique only on the morning of the surgery, thus making it a double-blinded study.

The approval of the institutional ethical committee was obtained (Army Hospital (R \& R), Delhi Cannt, approval number: 75/2015, date: 30.08 .2015$)$. Written informed consent was taken from all patients. Preoperative data recorded included age, sex, obstruction laterality, surgery indication, clinical abdominal findings, hemoglobin, blood urea, and serum creatinine levels. Anatomical radiological evaluation in ultrasonography and functional radiological evaluation in intravenous urography and Diethylene Triamine Penta Acetate (DTPA) renogram were also performed.

All surgical procedures were performed by consultants with equivalent experience. All surgeries were performed under general anesthesia in full lateral position after antibiotic prophylaxis administration (third-generation cephalosporin). Retro-grade pyelography was done before each surgery. Pneumoperitoneum was created using the veress needle technique. For RALP, one camera and two robotic arms of the four-arm da Vinci Si surgical system were used with one additional $10-\mathrm{mm}$ port for the assistant for suctioning or passage of suture materials and another 5-mm port for liver retraction (if required on the right side) (Figure 1). For CLP, one 10-mm camera port and two working ports were used (one $10 \mathrm{~mm}$ and another $5 \mathrm{~mm}$ ) (Figure 2). All patients underwent Anderson Hynes dismembered pyeloplasty using 4-0 vicryl sutures and intra-operatively antegrade Double-J stent (DJS) placement. Intra-operatively recorded parameters included the nature of the obstruction, surgery duration, blood loss, intraoperative complication, and instances involving conversion to an open approach. At the end of the procedure, a 20 French Foley catheter and 26 French

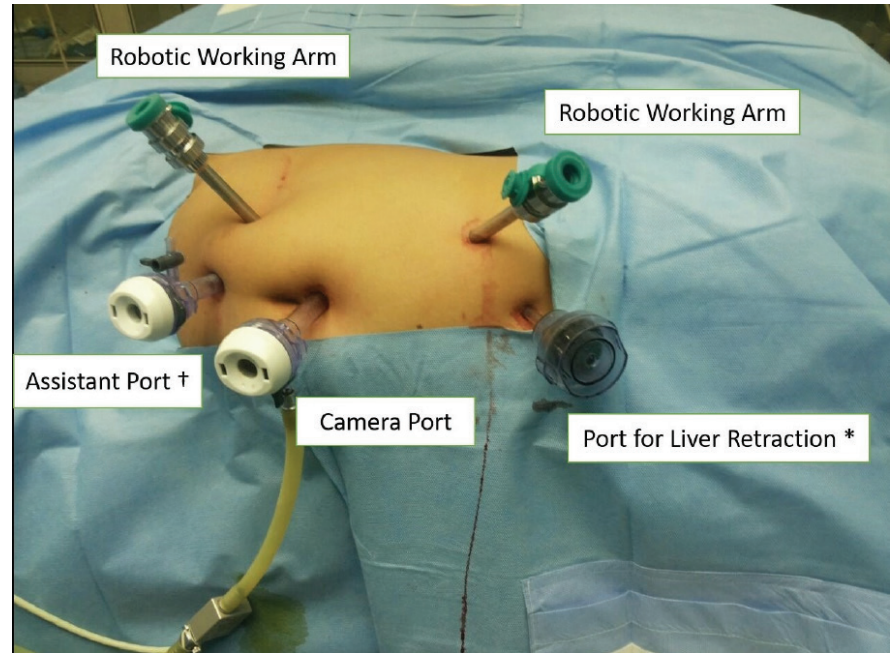

Figure 1. Port placement for right-sided robot-assisted pyeloplasty. *5$\mathrm{mm}$ port used for right-sided pyeloplasty to retract the lobe of the liver (If required). †10-mm assistant port used for suction and passage of suture material 
abdominal drains were placed in each case. Foley catheter was removed on a postoperative day (POD) 3 or 4 . The abdominal drain was subsequently removed, once the drain output was less than 30 milliliters per day for two consecutive days.

In the immediate postoperative period, patients were monitored for any surgical complication, pain severity, which was assessed using the visual analog score (VAS) for three consecutive days, analgesia requirement (injection of tramadol at $50 \mathrm{mg}$ intravenous was administered if patients had VAS of 2 or more on that day), hospital stay duration, drain placement duration, and time to return to daily activity.

Patients were followed up at 2 (for DJS removal) and 4 months (for DTPA scan) post-operatively. Additionally, a note was made of any postoperative complications.

Our study revealed a 100\% success rate in both groups, which was defined by resolution of patient's symptoms and demonstration of non-obstructed drainage on postoperative DTPA scan.

\section{Results}

This study included 58 patients with congenital UPJO (29 patients in each group) who underwent surgical management during the study period at our center. Complete data were available for all patients at the end of the study period. Basic demographic data are shown in Table 1. No statistically significant difference was found between the two groups concerning age, sex distribution, and obstruction laterality. Our study revealed a marginally higher percentage of patients who presented with symptoms in the CLP group compared to the RALP group; however, this difference also was statistically insignificant.

Among the intraoperative variables (Table 1), crossing vessel was relatively more frequently seen in the RALP group, but

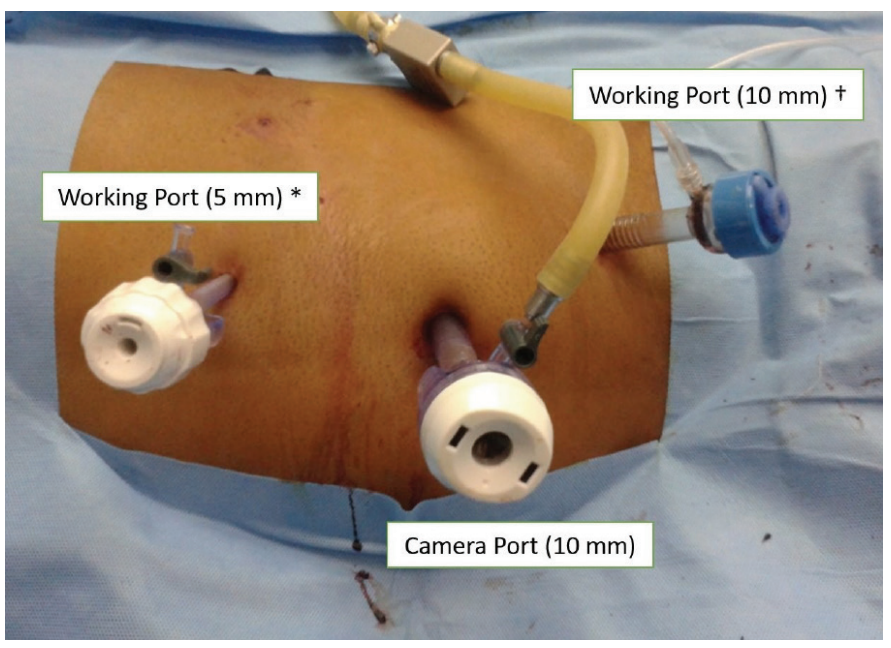

Figure 2. Port placement for left-sided laparoscopic pyeloplasty. ${ }^{*} 5$-mm port used by the surgeon for holding tissue. †10-mm port used for passage of needle with suture material, suction, etc. generally, aperistaltic/stenotic segment was noted as the most common etiology of obstruction in both groups. This variable was statistically insignificant in our study. The mean total operative time in the CLP group, which included time for pneumo-peritoneum creation, port placement, and surgical procedure, was noted as $34.28 \mathrm{~min}$ more than the RALP group, which includes time for pneumo-peritoneum creation, port placement, docking time, console time, and undocking time. Similarly, the total blood loss in the CLP group was noted as $9.2 \mathrm{~mL}$ more than the RALP group. Reduction in both intraoperative time and blood loss was statistically significant and in favor of the RALP group. No intraoperative complications or conversion to an open approach was noted in either of the groups.

Among the postoperative variables (Table 2), the VAS score on POD $1 / 2 / 3$, number of days of analgesia requirement, days of abdominal drain placement, hospitalization duration, and days required to return to daily activity were comparable and statistically insignificant between both groups. In both groups, no postoperative complications were noted, and all 29 cases in both groups demonstrated a non-obstructed flow pattern on follow-up with DTPA scan after 4 months.

\section{Discussion}

One of the most significant advances in the surgical field of the twenty-first century has been the introduction of laparoscopic surgery. Compared to open surgery, the major advantages of laparoscopic surgery include better cosmetics, lower

\begin{tabular}{|c|c|c|c|}
\hline Characteristics & CLP & RALP & p-value \\
\hline Number of patients & 29 & 29 & - \\
\hline Age in years & $35.44+10.25$ & $33.96+8.85$ & 0.58 \\
\hline Sex (female/male) & $13 / 16$ & $10 / 19$ & 0.38 \\
\hline Side (left/right) & $13 / 16$ & $17 / 12$ & 0.25 \\
\hline \multicolumn{4}{|l|}{ Presentation } \\
\hline Incidentally detected & 7 & 8 & 0.73 \\
\hline Symptomatic (Pain) & 22 & 21 & 0.73 \\
\hline \multicolumn{4}{|l|}{ Intraoperative finding } \\
\hline Crossing vessel & 8 & 10 & 0.52 \\
\hline $\begin{array}{l}\text { Aperistaltic/stenotic } \\
\text { segment }\end{array}$ & 21 & 19 & 0.52 \\
\hline $\begin{array}{l}\text { Total operative time } \\
\text { (minutes) }\end{array}$ & $148.56+15.15$ & $114.28+12.98$ & 0.001 \\
\hline $\begin{array}{l}\text { Total blood loss } \\
\text { (milliliter) }\end{array}$ & $68.4+7.86$ & $59.2+9.8$ & 0.001 \\
\hline Intra-op complications & Nil & Nil & - \\
\hline
\end{tabular}




\begin{tabular}{|l|l|l|l|}
\hline \multicolumn{4}{|l|}{ Table 2. Postoperative comparison } \\
\hline Characteristics & CLP & RALP & p-value \\
\hline VAS on post op day 1 & $4.86+0.33$ & $4.66+0.51$ & 0.11 \\
\hline VAS on post op day 2 & $2.84+0.40$ & $2.78+0.32$ & 0.56 \\
\hline VAS on post op day 3 & $1.26+0.41$ & $1.14+0.36$ & 0.28 \\
\hline Days analgesia Given & $2.08+0.27$ & $2.04+0.20$ & 0.56 \\
\hline Days drain kept & $4.16+0.62$ & $3.96+0.67$ & 0.28 \\
\hline Total hospitalization (Days) & $5.16+0.85$ & $4.96+0.88$ & 0.42 \\
\hline Return to daily activity (Days) & $20.4+2.6$ & $20.2+2.4$ & 0.8 \\
\hline Post op complication & Nil & Nil & - \\
\hline Success of surgery & $100 \%$ & $100 \%$ & - \\
\hline $\begin{array}{l}\text { CLP: Conventional laparoscopic pyeloplasty, RALP: } \\
\text { pyeloplasty }\end{array}$ & Robot-assisted laparoscopic \\
\hline
\end{tabular}

postoperative pain, reduced intraoperative blood loss, reduced hospital stay duration, and early return to daily activities despite having similar functional and oncological outcomes. A few of the major limitations of laparoscopic surgery are counter-intuitive and scaled-up movements, ergonomically tiring for surgeons, and lack of three-dimensional vision. All these ultimately lead to a steep learning curve.

With the introduction of robotic platforms in urology since the early 2000s, many of these limitations have been overcome. The major advantages of robotic surgery compared to laparoscopy include three-dimensional vision, elimination of tremors, and a better range of movements. With the wider availability of robotic devices, an increasing number of procedures, which were initially done using laparoscopy, are now done using robotic platforms worldwide with excellent outcomes. However, the limitations with robotic surgery include the associated higher costs and requirement of larger space in the operating room.

A recent systematic review and meta-analysis by Autorino et al. (4) confirmed that laparoscopy represents an effective and less invasive technique of pyeloplasty, but RALP is likely to emerge as the new minimally invasive standard of care wherever robotic technology is available due to its precise suturing and shorter learning curve. Previous studies compared RALP with CLP and revealed advantages in RALP in respect of total operative time, intraoperative blood loss, and length of hospitalization but revealed comparable results for postoperative complication and success rate (5-7).

A statistically significant difference was found in the mean total operative time of 34.28 min favoring the RALP group in our study. This finding was found consistent with other studies in the literature. Hemal et al. (6) (98 $\mathrm{min}$ in RALP vs. $145 \mathrm{~min}$ in CLP), Kumar and Nayak (8) (129 min in RALP vs. $150 \mathrm{~min}$ in CLP), and Pahwa et al. (9) (141.73 min in RALP vs. 191.56 min in (LP) also demonstrated statistically significant reduction favoring the RALP group in their respective studies. The reduced operative time in the RALP group is probably due to the better three-dimensional vision, tremor elimination, and better range of movements compared to the CLP group.

Our study revealed a statistically significant reduction in the total blood loss favoring the RALP group. Similarly, other studies in the literature have demonstrated similar findings. Hemal et al. (6) (40 mL in the RALP vs. $101 \mathrm{~mL}$ in the CLP group) and Pahwa et al. (9) $(46.37 \mathrm{~mL}$ in the RALP vs. $55.24 \mathrm{~mL}$ in the CLP group) demonstrated a statistically significant reduction in the total blood loss favoring the RALP group $(6,9)$. We feel that the reduced blood loss in the RALP group is associated with better vision, which results in more accurate dissection and precise hemostasis.

Additionally, in our study, we did not find any statistically significant difference between VAS scores between the two groups on POD 1/2/3. Pahwa et al. (9) demonstrated comparative postoperative pain scores between both the groups (4.77 in the CLP group and 4.16 in the RALP group). Likewise, Riachy et al. (10) involved the pediatric population and revealed comparable pain scores between the two groups.

Our study revealed a comparable mean number of days of keeping the abdominal drain following the surgery between the two groups. Consistent with our findings, Kumar and Nayak (8) (1.36 days for CLP vs. 1.58 days for RALP) and Pahwa et al. (9) (2.68 days for CLP vs. 2.03 days for RALP) also demonstrated comparable results. The requirement of longer duration of drain placement in our study (4.16 days in RALP versus 3.96 days in CLP) compared to the above-mentioned studies was due to the removal of drain only when the output was $<30 \mathrm{~mL}$ per day for 2 consecutive days (as per our institutional protocol), whereas the drains were removed once the output was $<50 \mathrm{~mL}$ over $24 \mathrm{~h}$ in both other studies.

Our study revealed a comparable number of hospitalization days between both groups. Similarly, Kumar and Nayak (8) (2.90 days for CLP vs. 2.89 days for RALP) and Pahwa et al. (9) (3 days for CLP vs. 2.45 days for RALP) found comparable results. Contrary to these findings, Braga et al. (11) revealed that the hospital stays in the RALP was significantly lesser than the CLP group, (weighted mean difference: -0.5 days; $95 \%$ confidence interval: $-0.6-0.4 ; p<0.01)$. The longer average duration of hospital stays in our study compared to the above-mentioned studies was attributable to the longer duration of abdominal drain placement in our patients.

The number of days required for the patient to return to daily activity was similarly comparable between the two groups in our study. We could not find any other study in literature which compared CLP and RALP concerning return to daily activity; however, Lasmar et al. (12) revealed that the time to return to normal activities following CLP ranged from 10 to 28 days (median 15 days). 
In both, groups in our study, no intraoperative or postoperative complication and requirement for conversion to open approach were found. Similarly, Gettman et al. (5) and Kumar and Nayak (8) demonstrated no intraoperative complications or requirement for conversion to an open approach. Pahwa et al. (9) revealed a complication rate of $11.4 \%$ in the CLP group and $8 \%$ in the RALP group, all of which were Clavien grade one or two, mainly prolonged drain output, UTI, and gut injury.

Our study had a 100\% success rate for both groups. Similarly, Kumar and Nayak (8) revealed a 100\% success rate for both groups in their study. Other studies revealed comparable outcomes between the two groups, with Gettman et al. (5), demonstrating no recurrence following the RALP group versus one recurrence following the CLP out of 30 cases in each group at 18 months postoperative follow-up and Pahwa et al. (9) demonstrated recurrence in one case each out of 30 in both groups. All these studies, like ours, confirm the comparable functional outcome for both modalities of treatment if meticulously performed.

\section{Study Limitatons}

The limitation of our study include the small sample size and the fact that the operating surgeons already had vast experience and were well versed in laparoscopic pyeloplasty at the start of the study, but due to the recent installation of Da Vinci Si Robotic surgical platform at our institute, the experience of the whole team in robotic procedures were limited, which could lead to a bias and inadvertently increased operative time in the RALP group at least during the first half of our study.

\section{Conclusion}

Finally, our study revealed a statistically significant reduction in both; however, the total operative time and intraoperative blood loss favoring RALP, of these only a reduction of intraoperative time by 34.28 min favoring RALP were clinically significant, whereas marginally lesser intraoperative blood loss by $9.2 \mathrm{~mL}$ favoring RALP was not clinically significant. All other postoperative surgical and functional parameters were comparable in both groups. Our study results were consistent with previously published studies as expected since, ultimately, robotic assistance refines laparoscopy in terms of precision of suture placement and tissue dissection and unchanged basic surgical approach.

\section{Ethics}

Ethics Committee Approval: The approval of the institutional ethical committee was obtained [Army Hospital (R \& R), Delhi Cannt, approval number: 75/2015, date: 30.08 .2015$]$.

Informed Consent: Written informed consent was taken from all patients.

Peer-review: Externally peer-reviewed.

\section{Authorship Contributions}

Surgical and Medical Practices: A.A.J., A.S.S., S.C.D., M.G., A.H., Concept: A.A.J., R.T., G.S., A.H., Design: A.A.J., S.C.D., R.T., G.S., A.H., Data Collection or Processing: A.A.J., R.T., M.G., G.S., N.S., Analysis or Interpretation A.A.J., A.S.S., S.C.D., R.T., M.G., G.S., N.S., Literature Search: A.A.J., S.C.D., M.G., A.H., Writing: A.A.J., A.S.S., S.C.D., R.T., M.G., G.S., A.H., N.S.

Conflict of Interest: No conflict of interest was declared by the authors.

Financial Disclosure: The authors declare that they have no relevant financial.

\section{References}

1. Baldwin DD, Dunbar JA, Wells N, McDougall EM. Single-center comparison of laparoscopic pyeloplasty, Acucise endopyelotomy, and open pyeloplasty. J Endourol 2003;17:155-160.

2. Winfield HN. Management of adult ureteropelvic junction obstruction--is it time for a new gold standard? J Urol 2006;176:866-867.

3. Warren J, da Silva V, Caumartin Y, Luke PP. Robotic renal surgery: The future or a passing curiosity? Can Urol Assoc J 2009;3:231-240.

4. Autorino R, Eden C, El-Ghoneimi A, Guazzoni G, Buffi N, Peters CA, Stein RJ, Gettman M. Robot-assisted and laparoscopic repair of ureteropelvic junction obstruction: a systematic review and meta-analysis. Eur Urol 2014;65:430-452.

5. Gettman MT, Peschel R, Neururer R, Bartsch G. A comparison of laparoscopic pyeloplasty performed with the daVinci robotic system versus standard laparoscopic techniques: initial clinical results. Eur Urol 2002;42:453-458.

6. Hemal AK, Mukherjee S, Singh K. Laparoscopic pyeloplasty versus robotic pyeloplasty for ureteropelvic junction obstruction: a series of 60 cases performed by a single surgeon. Can J Urol 2010;17:5012-5016.

7. Wang $\mathrm{F}, \mathrm{Xu} \mathrm{Y}$, Zhong $\mathrm{H}$. Robot-assisted versus laparoscopic pyeloplasty for patients with ureteropelvic junction obstruction: an updated systematic review and meta-analysis. Scand J Urol 2013;47:251-264.

8. Kumar R, Nayak B. Robotic versus conventional laparoscopic pyeloplasty: A single surgeon concurrent cohort review. Indian J Urol 2013;29:19-21.

9. Pahwa M, Pahwa AR, Girotra M, Abrahm RR, Kathuria S, Sharma A. Defining the pros and cons of open, conventional laparoscopy, and robot-assisted pyeloplasty in a developing nation. Adv Urol 2014;2014:850156.

10. Riachy E, Cost NG, Defoor WR, Reddy PP, Minevich EA, Noh PH. Pediatric standard and robot-assisted laparoscopic pyeloplasty: a comparative single institution study. J Urol 2013;189:283-287.

11. Braga LH, Pace K, DeMaria J, Lorenzo AJ. Systematic review and metaanalysis of robotic-assisted versus conventional laparoscopic pyeloplasty for patients with ureteropelvic junction obstruction: effect on operative time, length of hospital stay, postoperative complications, and success rate. Eur Urol 2009;56:848-857.

12. Lasmar MT, Castro HA Jr, Vengjer A, Guerra FA, Souza EA, Rocha LM. Transperitoneal laparoscopic pyeloplasty: Brazilian initial experience with 55 cases. Int Braz J Urol 2010;36:678-684. 